\title{
Oscillation of nonlinear neutral delay differential equations with applications
}

\author{
by Wan-Tong Li (Lanzhou) and S. H. SAKer (Mansoura)
}

\begin{abstract}
We consider nonlinear neutral delay differential equations with variable coefficients. Finite and infinite integral conditions for oscillation are obtained. As an example, the neutral delay logistic differential equation is discussed.
\end{abstract}

1. Introduction. A neutral delay differential equation is a differential equation in which the highest order derivative of the unknown function appears both with and without delays. The study of the asymptotic and oscillatory behavior of solutions of neutral differential equations is of importance in applications. This is due to the fact that such equations appear in various phenomena including networks containing lossless transmission lines (as in high-speed computers where such lines are used to interconnect switching circuits), in the study of vibrating masses attached to an elastic bar, as the Euler equations for the minimization of functionals involving a time delay in some variational problems, in the theory of automatic control and in neuromechanical systems in which inertia plays an important role (see Hale [11], Driver [3], Brayton and Willoughby [2], Popov [26] and Boe and Chang [1] and references cited therein). The construction of these models using delays has been paralleled by mathematical investigation of nonlinear equations.

Many authors have considered linear neutral delay differential equations and established sufficient conditions for oscillation of all solutions. We refer to the articles $[6,7,28,17,20,22,27,31]$ and the references cited therein.

In recent years there has been much research activity concerning the linearized oscillation theory for nonlinear neutral delay differential equations,

2000 Mathematics Subject Classification: 34K11, 34K40.

Key words and phrases: oscillation, nonlinear neutral delay differential equations, neutral logistic equation.

Research of W. T. Li supported by NNSF of China and the Foundation for University Key Teachers of the Ministry of Education of China. 
which is in some sense parallel to the so-called linearized stability theory (see $[5,8,19,25,29])$.

In this paper we consider the first-order nonlinear neutral delay differential equation with variable coefficients

$$
\frac{d}{d t}[x(t)-P(t) x(t-\tau)]+Q(t) f(x(t-\sigma))=0,
$$

where

$$
\begin{gathered}
0<P(t)<1, \sigma, \tau \text { are positive constants, } Q \in C\left[\left[t_{0}, \infty\right), \mathbb{R}^{+}\right], \\
f \in C[\mathbb{R}, \mathbb{R}], \quad u f(u)>0 \quad \text { for } u \neq 0,
\end{gathered}
$$

and there exists a positive number $\delta$ such that

$$
\begin{cases}f(u) \leq u & \text { for } u \in[0, \delta] \\ f(u) \geq u & \text { for } u \in[-\delta, 0]\end{cases}
$$

and

$$
\lim _{u \rightarrow 0} \frac{u}{f(u)}=\beta>0
$$

or the more general one,

$$
\frac{d}{d t}\left[x(t)-\sum_{i=1}^{n} P_{i}(t) x\left(t-\tau_{i}\right)\right]+\sum_{j=1}^{m} Q_{j}(t) f\left(x\left(t-\sigma_{j}\right)\right)=0,
$$

where $\tau_{i}, \sigma_{j}, P_{i}, Q_{j}$ and $f$ satisfy the same assumptions as $\tau, \sigma, P, Q$ and $f$ for $i=1, \ldots, n$ and $j=1, \ldots, m$.

Our aim in this paper is to give some finite and infinite integral sufficient conditions for oscillation of solutions of (1) and (6). In Section 2, we present some lemmas which will be used in the proofs of our main results. In Section 3, we give oscillation criteria for (1). In Section 4 the neutral delay logistic differential equation is considered to illustrate our results.

Let $\gamma=\max \{\sigma, \tau\}$ and let $t_{1} \geq t_{0}$. By a solution of $(1)$ on $\left[t_{1}, \infty\right)$ we mean a function $x \in C\left[\left[t_{1}-\gamma, \infty\right), \mathbb{R}\right]$ such that $x(t)-P(t) x(t-\tau)$ is continuously differentiable for $t_{1} \geq t_{0}$ and (1) is satisfied.

Let $t_{1} \geq t_{0}$ be a given initial point and let $\phi \in C\left[\left[t_{1}-\gamma, \infty\right), \mathbb{R}\right]$ be a given initial function. Then by the step-by-step method one can see that (1) has a unique solution on $\left[t_{1}, \infty\right)$ satisfying the initial condition

$$
x(t)=\phi(t) \quad \text { for }-\gamma \leq t \leq t_{1} .
$$

As usual, when we say that every solution of (1) oscillates we mean that for every initial point $t_{1} \geq t_{0}$ and for every initial function $\phi \in$ $C\left[\left[t_{1}-\gamma, \infty\right), \mathbb{R}\right]$, the unique solution of (1) with (7) has arbitrarily large zeros. Otherwise the solution is called nonoscillatory.

In what follows, when we write a functional inequality we will assume that it holds for all sufficiently large values of $t$. 
2. Some lemmas. In this section we present some lemmas that are used in the proofs of our main results.

Lemma 2.1 [10, Lemma 1.5.4]. Let $a \in(-\infty, 0), \tau \in(0, \infty), t_{0} \in \mathbb{R}$ and suppose that $x \in C\left[\left[t_{0}, \infty\right), \mathbb{R}\right]$ satisfies the inequality

$$
x(t) \leq a+\max _{t-\tau \leq s \leq t} x(s) \quad \text { for } t \geq t_{0} .
$$

Then $x(t)$ cannot be a nonnegative function.

Lemma 2.2 [10, Lemma 1.5.5]. Let $F, G, P \in C\left[\left[t_{0}, \infty\right), \mathbb{R}\right]$, and $c \in$ $(0, \infty)$ be such that

$$
F(t)=G(t)-P(t) G(t-c), \quad t \geq t_{0}+c .
$$

Assume that

$$
F(t)>0 \quad \text { and } \quad G(t)>0 \quad \text { for } \quad t \geq t_{0}, \quad \lim _{t \rightarrow \infty} F(t)=0,
$$

and $0<P(t)<1$. Then $\lim _{t \rightarrow \infty} G(t)=0$.

Lemma 2.3 Assume that (2) and (3) are satisfied. Set

$$
z(t)=x(t)-P(t) x(t-\tau),
$$

where $x(t)$ is an eventually positive solution of (1). Then $z(t)$ is an eventually nonincreasing positive function, $\lim _{t \rightarrow \infty} z(t)=0$, and $\lim _{t \rightarrow \infty} x(t)=0$.

Proof. Since $x(t)$ is an eventually positive solution of (1), we have

$$
z^{\prime}(t) \leq-Q(t) f(x(t-\sigma)) .
$$

Then from (2) and (3) we have

$$
z^{\prime}(t) \leq-Q(t) f(x(t-\sigma) \leq 0,
$$

where $z^{\prime}(t)=d z(t) / d t$. This shows that $z(t)$ is nonincreasing. Now we show that $z(t)$ is positive. For otherwise there exists a $t_{2} \geq t$ such that $z\left(t_{2}\right)<0$. Because $\dot{z}(t) \leq 0$ for $t \geq t_{1}+\varrho$ and $\dot{z}(t) \neq 0$ on $\left[t_{1}+\varrho, \infty\right)$ there exists $t_{3} \geq t_{2}$ such that $z(t) \leq z\left(t_{3}\right)$ for $t \geq t_{3}$. Then from (8) it follows that for $t \geq t_{3}$

$$
x(t)=z(t)+P(t) x(t-\tau) \leq z\left(t_{3}\right)+P(t) x(t-\tau) .
$$

Hence

$$
x(t) \leq z\left(t_{2}\right)+\max _{t-\varrho \leq s \leq t} x(s) P(t) .
$$

From (2) we have

$$
x(t) \leq z\left(t_{2}\right)+\max _{t-\varrho \leq s \leq t} x(s) \quad \text { for } t \geq t_{3} .
$$

Thus by Lemma $2.1, x(t)$ cannot be a non-negative function on $\left[t_{3}, \infty\right)$, and this is a contradiction. Hence $z(t)$ is non-increasing and positive. 
Now we show that $x(t)$ is bounded. Otherwise there exists a sequence $\left\{t_{n}\right\}$ such that $\lim _{n \rightarrow \infty} t_{n}=\infty, \lim _{n \rightarrow \infty} x\left(t_{n}\right)=\infty$ and $x\left(t_{n}\right)=\max _{s \leq t_{n}} x(s)$. From (8) we have

$$
z\left(t_{n}\right)=x\left(t_{n}\right)-P\left(t_{n}\right) x\left(t_{n}-\tau\right) .
$$

Hence by (2) we obtain

$$
z\left(t_{n}\right) \geq x\left(t_{n}\right)\left[1-P\left(t_{n}\right)\right] \rightarrow \infty \quad \text { as } n \rightarrow \infty,
$$

which contradicts (9). Then by (8) and (9) we see that $z(t)$ is also bounded, and

$$
\lim _{t \rightarrow \infty} z(t)=k \in[-\infty, \infty) .
$$

If $k=-\infty$, then there is a $t_{1} \geq t_{0}$ such that $z(t)<0$ for $t \geq t_{1}$. From (8) we have $\lim \sup _{t \rightarrow \infty} x(t)=+\infty$, which contradicts the fact that $x(t)$ is bounded. Thus $k \neq-\infty$.

From (9) and (10) it follows that the function $z(t)$ is monotonic. By integrating both sides of (9) from $t_{1}$ to $\infty$ for $t_{1}$ sufficiently large, we obtain

$$
k-z\left(t_{1}\right)=-\int_{t_{1}}^{\infty} Q(t) f(x(t-\sigma)) d t .
$$

We claim that

$$
\liminf _{t \rightarrow \infty} x(t)=0 .
$$

Otherwise there exist a positive constant $c$ and a $t_{2} \geq t_{1}$ such that $x(t) \geq c$ for $t \geq t_{2}$. Then from (2) and (3) and for $t$ sufficiently large, $Q(t) f(x(t-\sigma))$ is bounded from below by a positive constant. This contradicts (11) and so (12) holds.

Let $\left\{t_{k}\right\}$ be a sequence of points such that

$$
\lim _{k \rightarrow \infty} t_{k}=\infty \text { and } \lim _{k \rightarrow \infty} x\left(t_{k}\right)=0 .
$$

From (8) we see that

$$
z\left(t_{k}\right) \leq x\left(t_{k}\right) \rightarrow 0 \quad \text { as } k \rightarrow \infty
$$

and also

$$
z\left(t_{k}+\tau\right) \geq-P\left(t_{k}+\tau\right) x\left(t_{k}\right) \rightarrow 0 \quad \text { as } k \rightarrow \infty .
$$

As $z(t)$ is positive and monotonic, it follows that $\lim _{t \rightarrow \infty} z(t)=0$. Then by Lemma 2.2 we see that $\lim _{t \rightarrow \infty} x(t)=0$. This completes the proof.

3. Oscillation criteria. In this section we give finite and infinite integral conditions for oscillation of solutions of (1). 
Theorem 3.1. Assume that (2)-(5) hold. Then the conditions

$$
\liminf _{t \rightarrow \infty} \int_{t-\sigma}^{t}\left[Q_{1}(s) P(t-\sigma)+Q_{1}(s)\right] d s>\frac{1}{e}
$$

and

$$
\limsup _{t \rightarrow \infty} \int_{t-\sigma}^{t}\left[Q_{1}(s) P(t-\sigma)+Q_{1}(s)\right] d s>1
$$

each imply that every solution of (1) oscillates, where

$$
Q_{1}(t)=\frac{1}{\beta} Q(t), \quad \text { and } \quad \tau \geq \sigma .
$$

Proof. Without loss of generality we assume that (1) has an eventually positive solution $x(t)$ (the case that $x(t)$ is negative is similar and will be omitted). Suppose $x(t)>0$ and $x(t-\sigma)>0$ for $t \geq t_{0}$. Then from Lemma 2.3 we have $\lim _{t \rightarrow \infty} x(t)=0$. By (5) we have

$$
\lim _{u \rightarrow 0} \frac{u}{f(u)}=\beta>0 .
$$

Let $\varepsilon \in(0, \beta)$. Then there exists a $T_{\varepsilon}$ such that for $t \geq T_{\varepsilon}, x(t-\sigma)>0$ and $f(x(t-\sigma)) \geq x(t-\sigma) /(\beta-\varepsilon)$.

Set $z(t)$ as in (8). Then from (1) and Lemma 2.3, $z(t)$ is a positive function, $x(t)>z(t)$ and

$$
z^{\prime}(t)+Q(t) \frac{x(t-\sigma)}{\beta-\varepsilon} \leq 0, \quad t \geq T_{\varepsilon} .
$$

Hence,

$$
z^{\prime}(t)+Q_{1}(t) x(t-\sigma) \leq 0, \quad t \geq T_{\varepsilon},
$$

where $Q_{1}(t)=Q(t) / \beta$. Then from (8),

$$
\begin{aligned}
z^{\prime}(t) & \leq-Q_{1}(t) z(t-\sigma)-Q_{1}(t) P(t-\sigma) x(t-\tau-\sigma), \quad t \geq T_{\varepsilon}, \\
& \leq-Q_{1}(t) z(t-\sigma)+\frac{Q_{1}(t)}{Q_{1}(t-\tau)} P(t-\sigma) z^{\prime}(t-\tau) .
\end{aligned}
$$

Hence $z(t)$ is positive and satisfies the delay differential inequality

$$
z^{\prime}(t)-\frac{Q_{1}(t)}{Q_{1}(t-\tau)} P(t-\sigma) z^{\prime}(t-\tau)+Q_{1}(t) z(t-\sigma) \leq 0 .
$$

Set

$$
\lambda(t)=-\frac{z^{\prime}(t)}{z(t)} .
$$

Then (19) becomes 


$$
\begin{aligned}
\lambda(t) \geq & \lambda(t-\tau) \frac{Q_{1}(t)}{Q_{1}(t-\tau)} P(t-\sigma) \\
& \times \exp \left(\int_{t-\tau}^{t} \lambda(s) d s\right)+Q_{1}(t) \exp \left(\int_{t-\sigma_{1}}^{t} \lambda(s) d s\right) .
\end{aligned}
$$

It is obvious that $\lambda(t)>0$ for $t \geq t_{0}$. From (21) we have $\lambda(t) \geq Q_{1}(t)$, thus

$$
\lambda(t) \geq Q_{1}(t) P(t-\sigma) \exp \left(\int_{t-\tau}^{t} \lambda(s) d s\right)+Q_{1}(t) \exp \left(\int_{t-\sigma_{1}}^{t} \lambda(s) d s\right) .
$$

Then from (22) and (20) one can see that $z(t)$ is a positive solution of the delay differential inequality

$$
z^{\prime}(t)+Q_{1}(t) P(t-\sigma) z(t-\tau)+Q_{1}(t) z(t-\sigma) \leq 0 .
$$

As $z^{\prime}(t) \leq 0$ and $\tau \geq \sigma$, we see that

$$
z^{\prime}(t)+\left[Q_{1}(t) P(t-\sigma)+Q_{1}(t)\right] z(t-\sigma) \leq 0 .
$$

Then by Corollary 3.2.2 of [10] the delay differential equation

$$
z^{\prime}(t)+\left[Q_{1}(t) P(t-\sigma)+Q_{1}(t)\right] z(t-\sigma)=0
$$

has an eventually positive solution as well. However, it is well known that (14) and (15) each imply that (23) has no eventually positive solution (see, for example, [10, p. 46, Theorem 2.3.3] and [10, p. 78, Theorem 3.4.3]). This is a contradiction and so the proof is complete.

REMARK 1. It is clear that every solution of (1) oscillates when (23) has no eventually positive solution. The problem how to fill the gap between the conditions (13) and (14) for the equation (23) has recently been investigated by several authors. Taking the results of $[4,14,18,23,24,30]$ respectively into account and the fact that every solution of (1) oscillates when (23) has no eventually positive solution we obtain the following applications, where we set

$$
\bar{Q}(t)=Q_{1}(t) P(t-\sigma)+Q_{1}(t) .
$$

Corollary 3.1. Assume that (2)-(5) hold,

and

$$
\liminf _{t \rightarrow \infty} \int_{t-\sigma}^{t} \bar{Q}(s) d s=k \leq \frac{1}{e}
$$

$$
\limsup _{t \rightarrow \infty} \int_{t-\sigma}^{t} \bar{Q}(s) d s>\frac{\ln (\lambda)+1}{\lambda}
$$

where $\lambda$ is the smaller solution of the transcendental equation

$$
\lambda=e^{\lambda k} .
$$

Then every solution of (1) oscillates. 
Corollary 3.2. Assume that (2)-(5) hold,

$$
k=\liminf _{t \rightarrow \infty} \int_{t-\sigma}^{t} \bar{Q}(s) d s, \quad L=\limsup _{t \rightarrow \infty} \int_{t-\sigma}^{t} \bar{Q}(s) d s,
$$

$L<1$ and $0<k \leq 1 /$ e. Then every solution of (1) oscillates if

$$
L>\frac{\ln (\lambda)+1}{\lambda}-\frac{1-k-\sqrt{1-k-k^{2}}}{2},
$$

where $\lambda$ is the smaller root of (24).

Corollary 3.3. Assume that (2)-(5) hold and

$$
\sum_{i=1}^{\infty}\left[\int_{t_{i-1}}^{t_{i}} \bar{Q}(s)-\frac{1}{e}\right] d s=\infty
$$

Then every solution of (1) oscillates.

Corollary 3.4. Assume that (2)-(5) hold,

$$
\int_{t-\sigma}^{t} \bar{Q}(s) d s \geq \frac{1}{e}
$$

and

$$
\int_{t_{0}+\sigma}^{\infty} \bar{Q}(t)\left[\exp \left(\int_{t-\sigma}^{t} \bar{Q}(s) d s-\frac{1}{e}\right)-1\right] d s=\infty .
$$

Then every solution of (1) oscillates.

Corollary 3.5. Assume that (2)-(5) hold,

$$
\int_{t-\sigma}^{t} \bar{Q}(s) d s \geq \frac{1}{e}
$$

and

$$
\int_{t_{0}+n \sigma}^{\infty} \bar{Q}(t)\left[e^{n-1} \bar{Q}_{n}(t)-\frac{1}{e}\right] d t=\infty
$$

Then every solution of (1) oscillates. Here

$$
\begin{aligned}
\bar{Q}_{1}(t) & =\int_{t-\sigma}^{t} \bar{Q}(s) d s, & t \geq t_{0}+\sigma, \\
\bar{Q}_{k+1}(t) & =\int_{t-\sigma}^{t} \bar{Q}(s) \bar{Q}_{k}(s) d s, & t \geq t_{0}+(k+1) \sigma .
\end{aligned}
$$


Corollary 3.6. Assume that (2)-(5) hold,

$$
\limsup _{t \rightarrow \infty} \int_{t}^{t+\sigma} \bar{Q}(s) d s>0 \quad \text { for } t \geq t_{0} \text { for some } t_{0}>0,
$$

and

$$
\int_{t_{0}}^{\infty} \bar{Q}(t) \ln \left[e \int_{t}^{t+\sigma} \bar{Q}(s) d s\right] d t=\infty .
$$

Then every solution of (1) oscillates.

Theorem 3.2. Assume that (2)-(5) hold. Then the conditions

$$
\liminf _{t \rightarrow \infty} \int_{t-\sigma}^{t} Q_{1}(t) P(t-\sigma)>\frac{1}{e}
$$

and

$$
\limsup _{t \rightarrow \infty} \int_{t-\sigma}^{t} Q_{1}(t) P(t-\sigma) d s>1
$$

each imply that every solution of (1) oscillates.

Proof. Without loss of generality, assume that (1) has an eventually positive solution $x(t)$. Let $z(t)$ be defined by (8). Then from (23) one can see that $z(t)$ is a positive solution of the inequality

$$
z^{\prime}(t)+Q_{1}(t) P(t-\sigma) z(t-\sigma) \leq 0 .
$$

Therefore by Corollary 3.2.2 of [10] the delay differential equation

$$
z^{\prime}(t)+Q_{1}(t) P(t-\sigma) z(t-\sigma)=0
$$

has an eventually positive solution as well. But it is well known that (25) and (26) each imply that (28) has no eventually positive solution. This is a contradiction and so the proof is complete.

Theorem 3.3. Assume that (2)-(5) hold. Then the conditions

$$
\liminf _{t \rightarrow \infty} \int_{t-\sigma}^{t} Q_{1}(s) d s>\frac{1}{e}
$$

and

$$
\limsup _{t \rightarrow \infty} \int_{t-\sigma}^{t} Q_{1}(s) d s>1
$$

each imply that every solution of (1) oscillates. 
Proof. Assume that (1) has an eventually positive solution $x(t)$. Let $z(t)$ be defined by (8). Then from (23) one can see that

$$
z^{\prime}(t)+Q_{1}(t) z(t-\sigma) \leq 0 .
$$

Therefore by Corollary 3.2.2 of [10] the delay differential equation

$$
z^{\prime}(t)+Q_{1}(t) z(t-\sigma)=0
$$

has an eventually positive solution as well. But it is well known that (29) and (30) each imply that (32) has no eventually positive solution. This is a contradiction and so the proof is complete.

Theorem 3.4. Assume that (2)-(5) hold. Then the conditions

$$
\liminf _{t \rightarrow \infty} \int_{t-\sigma}^{t}\left[Q_{1}(s) P(s-\sigma) P(s-\tau-\sigma)+Q_{1}(s)\right] d s>\frac{1}{e}
$$

and

$$
\limsup _{t \rightarrow \infty} \int_{t-\sigma}^{t}\left[Q_{1}(s) P(s-\sigma) P(s-\tau-\sigma)+Q_{1}(s)\right] d s>1
$$

each imply that every solution of (1) oscillates.

Proof. Assume that (1) has an eventually positive solution $x(t)$. From (21) it is obvious that $\lambda(t)>0$ for $t \geq t_{0}$, and $\lambda(t) \geq Q_{1}(t)$. Then $\lambda(t-\tau) \geq$ $Q_{1}(t-\tau)$, and so

$$
\lambda(t) \geq Q_{1}(t) P(t-\sigma) \exp \left(\int_{t-\tau}^{t} \lambda(s) d s\right)+Q_{1}(t) \exp \left(\int_{t-\sigma}^{t} \lambda(s) d s\right),
$$

which guarantees that $\lambda(t) \geq Q_{1}(t) P(t-\sigma)$. Thus

$$
\lambda(t-\tau) \geq Q_{1}(t-\tau) P(t-\tau-\sigma) .
$$

From (32) we have

$$
\begin{aligned}
\lambda(t) \geq & Q_{1}(t) P(t-\sigma) P(t-\tau-\sigma) \\
& \times \exp \left(\int_{t-\tau}^{t} \lambda(s) d s\right)+Q_{1}(t) \exp \left(\int_{t-\sigma}^{t} \lambda(s) d s\right)
\end{aligned}
$$

and therefore $z(t)$ satisfies the inequality

$$
z^{\prime}(t)+\left[Q_{1}(t) P(t-\sigma) P(t-\tau-\sigma)+Q_{1}(t)\right] z(t-\sigma) \leq 0 .
$$

Then by Corollary 3.2.2 of [10] the delay differential equation

$$
z^{\prime}(t)+\left[Q_{1}(t) P(t-\sigma) P(t-\tau-\sigma)+Q_{1}(t)\right] z(t-\sigma)=0
$$

has an eventually positive solution as well. But it is well known that (33) and (34) each imply that (37) has no eventually positive solution. Thus every solution of (1) oscillates. 
By applying the above corollaries to the equations (37) we can obtain sufficient conditions for oscillation of all solutions of (1). Their statements are omitted here.

REMARK 2. For the general equation

$$
\frac{d}{d t}\left[x(t)-P \sum_{i=1}^{n} P_{i}(t) x\left(t-\tau_{i}\right)\right]+\sum_{i=1}^{n} Q_{i}(t) x\left(t-\sigma_{i}\right)=0, \quad t \geq t_{0},
$$

the analogues of the above results are also true. We omit the details.

\section{Oscillation in a nonautonomous neutral delay logistic equa-} tion. The scalar autonomous ordinary differential equation

$$
\dot{N}(t)=r N(t)\left[1-\frac{N(t)}{K}\right]
$$

is known as the logistic equation in mathematical ecology and it is a prototype in the modelling of the dynamics of single-species population systems whose biomass or density is described by a differentiable function $N(t)$. The constant $r$ is called the growth rate and $K$ is called the carrying capacity of the habitat. Hutchinson [13] suggested the following modification:

$$
\dot{N}(t)=r N(t)\left[1-\frac{N(t-\tau)}{K}\right] .
$$

Equation (38) is commonly known as the "delay equation" and has been extensively investigated by numerous authors (see for example Wright [31], Kakutani and Markus [16] and Jones [15]). Hegazi and Saker [12] considered the non-autonomous delay logistic and food limited equations and presented infinite integral conditions for oscillations.

Györi [9] considered the neutral delay logistic equation with constant coefficients of the form

$$
\dot{N}(t)=N(t)\left[r\left(1-\frac{N(t-\tau)}{K}\right)+c \dot{N}(t-\tau)\right],
$$

and established oscillation criteria for all positive solutions.

The effects of varying environment are often important in dynamical nature of populations. Then we consider the nonautonomous neutral delay equation

$$
\dot{N}(t)=N(t)\left[r(t)\left(1-\frac{N(t-\sigma)}{K}\right)+c(t) \dot{N}(t-\tau)\right]
$$

where

$$
r, c \in C\left[\left[t_{0}, \infty\right), \mathbb{R}^{+}\right], \quad K, \tau, \sigma \in(0, \infty),
$$


$r(t)$ is the growth rate function, $K$ is the carrying capacity of the environment, and $c(t)$ is the growth rate function associated with the growth rate at time $t-\tau$.

With (38) one associates an initial condition of the form

$$
N(t)=\phi(t) \quad \text { for }-\gamma \leq t \leq 0, \quad \phi \in C\left[[-\tau, 0], \mathbb{R}^{+}\right], \quad \phi(0)>0,
$$

where $\gamma=\max \{\tau, \sigma)$. Then by the step-by-step method, the initial value problem (40) and (42) has a unique solution $N(t)$ for $t \geq 0$. We will only consider those solutions $N(t)$ which are positive. Note that such solutions exist because if $\phi(0)>0$, then $N(t)>0$ for $t \geq 0$.

Theorem 4.1. Assume that (41) holds, $0<c(t)<1$, and

$$
\liminf _{t \rightarrow \infty} \int_{t-\sigma}^{t} r(s) d s>\frac{1}{e}
$$

Then every solution of (40) oscillates.

Proof. The change of variables $N(t)=K e^{x(t)}$ reduces (40) to the delay equation

$$
\frac{d}{d t}[x(t)-c(t) x(t-\tau)]+r(t)\left[e^{x(t-\sigma)}-1\right]=0 .
$$

Clearly, $N(t)$ oscillates about $K$ if and only if $x(t)$ oscillates about zero. From (44) we have

$$
\frac{d}{d t}[x(t)-c(t) x(t-\tau)]+r(t) f(x(t-\sigma))=0
$$

with

$$
f(u)=e^{u}-1
$$

It is clear that

$$
\begin{gathered}
f \in C[\mathbb{R}, \mathbb{R}], \quad u f(u)>0 \quad \text { for } u \neq 0, \\
\lim _{u \rightarrow 0} \frac{u}{f(u)}=1 .
\end{gathered}
$$

Then by Theorem 3.2 and the condition (43) every solution of (44) oscillates. Thus every positive solution of (40) oscillates about $K$.

REMARK 3. One can apply the above theorems and corollaries to obtain many sufficient conditions for oscillations. Also one can extend these results to the generalized neutral logistic equation

$$
\dot{N}(t)=\sum_{i=1}^{n} N(t)\left[r_{i}(t)\left(1-\frac{N\left(t-\sigma_{i}\right)}{K}\right)+c_{i}(t) N\left(t-\tau_{i}\right)\right] .
$$




\section{References}

[1] E. Boe and H. C. Chang, Dynamics of delayed systems under feedback control, Chem. Engrng. Sci. 44 (1989), 1281-1294.

[2] R. K. Brayton and R. A. Willoughby, On the numerical integration of a symmetric system of difference-differential equations of neutral type, J. Math. Anal. Appl. 18 (1967), 182-189.

[3] D. R. Driver, A mixed neutral system, Nonlinear Anal. 8 (1984), 155-158.

[4] A. Elbert and I. P. Stavroulakis, Oscillation and non-oscillation criteria for delay differential equations, Proc. Amer. Math. Soc. 124 (1995), 1503-1511.

[5] H. A. El-Morshedy and K. Gopalsamy, Nonoscillation, oscillation and convergence of a class of neutral equations, Nonlinear Anal. 40 (2000), 173-183.

[6] M. K. Grammatikopoulos, E. A. Grove and G. Ladas, Oscillation of first-order neutral delay differential equations, J. Math. Anal. Appl. 120 (1986), 510-520.

[7] M. K. Grammatikopoulos, Y. G. Sficas and G. Ladas, Oscillation and asymptotic behavior of neutral equations with variable coefficients, Rad. Mat. 2 (1986), 279-303.

[8] X. P. Guan, J. Yang and S. S. Cheng, Linearized comparison criteria for a nonlinear neutral differential equation, Ann. Polon. Math. 64 (1996), 161-173.

[9] I. Györi, Oscillation and comparison results in neutral differential equations and their applications to the delay logistic equation, Comput. Math. Appl. 18 (1989), 893-906.

[10] I. Györi and G. Ladas, Oscillation Theory of Delay Differential Equations with Applications, Clarendon Press, Oxford, 1991.

[11] J. K. Hale, Theory of Functional Differential Equations, Springer, New York, 1977.

[12] A. S. Hegazi and S. H. Saker, Oscillation of nonlinear delay differential equations, Internat. J. Differential Equations Appl., to appear.

[13] G. E. Hutchinson, Circular casual systems in ecology, Ann. New York Acad. Sci. 50 (1948), 221-240.

[14] J. Jaros and I. P. Stavroulakis, Oscillation tests for delay equations, Rocky Mountain J. Math., 29 (1999), 1-11.

[15] G. S. Jones, On the nonlinear differential-difference equation $f^{\prime}(x)=a f(x(-1))[1+$ $f(x)$ ], J. Math. Anal. Appl. 32 (1982), 440-469.

[16] S. Kakutani and L. Markus, On the nonlinear difference-differential equation $\dot{y}(t)=$ $[A-B y(t-\tau)] y(t)$, in: Contributions to the Theory of Nonlinear Oscillations, Vol. 4, Princeton Univ. Press, Princeton, NJ, 1958, 1-18.

[17] M. R. S. Kulenović, G. Ladas and A. Meimaridou, Necessary and sufficient condition for oscillations of neutral differential equations, J. Austral. Math. Soc. Ser. B 28 (1987), 362-375.

[18] M. K. Kwong, Oscillation of first order delay equations, J. Math. Anal. Appl. 159 (1991), 469-484.

[19] G. Ladas, Linearized oscillations for neutral equations, in: Differential Equations (Xanthi, 1987), Dekker, New York, 1989, 379-387.

[20] G. Ladas and Y. Sficas, Oscillations of neutral delay differential equations, Canad. Math. Bull. 29 (1986), 438-445.

[21] G. S. Ladde, V. Lakshmikantham and B. G. Zhang, Oscillation Theory of Differential Equations with Deviating Arguments, Dekker, New York, 1987.

[22] B. S. Lalli and B. G. Zhang, Oscillation of first-order neutral differential equations, Appl. Anal. 39 (1990), 265-274.

[23] B. Li, Oscillations of delay differential equations with variable coefficients, J. Math. Anal. Appl. 192 (1998), 312-321. 
[24] B. Li, Oscillation of first order delay differential equations, Proc. Amer. Math. Soc. 124 (1996), 3729-3737.

[25] H. J. Li, Qualitative analysis of positive solutions of first-order functional differential equations of neutral type, Nonlinear Anal. 39 (2000), 891-907.

[26] E. P. Popov, Automatic Regulation and Control, Nauka, Moscow, 1966 (in Russian).

[27] C. Qian, M. R. S. Kulenović and G. Ladas, Oscillation of neutral equations with variable coefficients, Rad. Mat. 5 (1989), 321-331.

[28] J. J. Sun and J. G. Wang, On a conjecture of Chen and Huang, ibid. 207 (1997), $61-72$.

[29] X. H. Tang, Oscillation of first-order nonlinear neutral delay differential equations, Hunnan Ann. Math. 16 (1996), 91-97.

[30] X. H. Tang and J. H. Shen, Oscillations of delay differential equations with variable coefficients, J. Math. Anal. Appl. 217 (1998), 32-42.

[31] E. M. Wright, A non-linear difference-differential equation, J. Reine Angew. Math. 194 (1955), 66-87.

[32] B. G. Zhang, Oscillation of first order neutral functional differential equations, J. Math. Anal. Appl. 139 (1989), 311-318.

Department of Mathematics

Lanzhou University

Lanzhou 730000, People's Republic of China

E-mail: wtli@lzu.edu.cn
Mathematics Department Faculty of Science Mansoura University Mansoura 35516, Egypt E-mail: shsaker@mum.mans.eun.eg

Reçu par la Rédaction le 9.10.2000

Révisé le 25.1.2001 